\title{
Injectable Cell Scaffold Restores Impaired Cell-Based Therapeutic Angiogenesis in Diabetic Mice with Hindlimb Ischemia
}

Koji Takeda ${ }^{1}$, Shinya Fukumoto ${ }^{* 2}$, Koka Motoyama ${ }^{1}$, Tomoaki Morioka ${ }^{1}$, Katsuhito Mori $^{1}$, Ken Kageyama ${ }^{3}$, Yukimasa Sakai ${ }^{3}$, Hideki Sato ${ }^{4}$, Masakazu Suzuki ${ }^{4}$, Hidenori Koyama $^{5}$, Tetsuo Shoji ${ }^{6}$, Eiji Ishimura ${ }^{7}$, Masanori Emoto ${ }^{1}$, Tsutomu Furuzono ${ }^{8}$, Koichi Nakajima $^{9}$, and Masaaki Inaba ${ }^{1}$

${ }^{1}$ Department of Metabolism, Endocrinology, and Molecular Medicine, ${ }^{2}$ Department of Premier Preventive Medicine, ${ }^{3}$ Department of Radiology, ${ }^{6}$ Department of Geriatrics and Vascular Medicine, ${ }^{7}$ Department of Nephrology, and ${ }^{9}$ Department of Immunology, Osaka City University Graduate School of Medicine, Osaka, Japan; ${ }^{4}$ Gunze limited, Osaka, Japan; ${ }^{5}$ Department of Internal Medicine, Division of Diabetes, Endocrinology and Metabolism, Hyogo College of Medicine, Hyogo, Japan; ${ }^{8}$ Department of Biomedical Engineering, School of Biology-Oriented Science and Technology, Kinki University, Wakayama, Japan.

${ }^{*}$ Correspondence should be addressed to:

Shinya Fukumoto, MD, PhD

Department of Premier Preventive Medicine,

Osaka City University Graduate School of Medicine

MedCity21, ABENO HARUKAS 21F, 1-1-43, Abenosuji, Abeno-ku, Osaka 545-6090, Japan 
Tel.: +81-6-6624-4010, Fax: +81-6-6624-8212

E-mail: sfukumoto@med.osaka-cu.ac.jp 


\begin{abstract}
The clinical success of cell-based therapeutic angiogenesis has been limited in diabetic patients with critical limb ischemia. We previously reported that an injectable cell scaffold (ICS), which is a nano-scaled hydroxyapatite (HAp)-coated polymer microsphere, enhances therapeutic angiogenesis. Subsequently, we developed a modified ICS for clinical use, measuring $50 \mu \mathrm{m}$ in diameter using poly(L-lactide-co-e-caprolactone) as a biodegradable polymer, which achieved appropriately accelerated absorption in vivo. The aim of the present study was to evaluate the effectiveness of this practical ICS in diabetic hindlimb ischemia.

Bone-marrow mononuclear cells (BMNCs) were intramuscularly injected, without or with a practical ICS, into the ischemic hindlimbs of mice (BMNCs or ICS + BMNCs group, respectively). Kaplan-Meier analysis demonstrated that the beneficial effects of BMNC transplantation for limb salvage after ischemic surgery were almost entirely abrogated in streptozotocin-induced diabetic mice. In contrast, injection of ICS + BMNCs revealed significant limb salvage in diabetic mice to a similar extent as in non-diabetic mice. The number of apoptotic transplanted BMNCs was 1.8 -fold higher in diabetic mice 10 days after transplantation compared to non-diabetic mice, while that in the ICS + BMNCs group was markedly lower (8.3\% of that in the BMNCs group) even in diabetic mice. The proangiogenic factors VEGF and FGF2, also known as antiapoptotic factors, mostly co-localized with transplanted GFP-positive BMNCs that were closely aggregated around the ICS in ischemic tissue.

In conclusion, the practical ICS significantly augmented cell-based therapeutic angiogenesis even in diabetic animals, through local accumulation of proangiogenic factors and antiapoptotic effects in transplanted cells.
\end{abstract}


Key Words: scaffold; diabetes; limb ischemia; therapeutic angiogenesis; cell transplantation; nanotechnology. 


\section{Introduction}

Patients with diabetes mellitus (DM) are at high risk of developing peripheral arterial disease (PAD) [1], and critical limb ischemia (CLI), the most advanced stage of $\mathrm{PAD}$, is associated with amputation and mortality [2]. PAD in DM patients is characterized by multiple diffuse arteriosclerotic lesions throughout the lower extremity artery. In particular, lesions that develop in the infra-popliteal artery can become severe occlusive lesions accompanied by calcification [3]. Therefore, DM patients with CLI cannot be treated by revascularization procedures such as bypass surgery or transluminal angioplasty, often leaving amputation as the only option [1].

In the past decade, we and other groups have reported the clinical effectiveness and safety of therapeutic angiogenesis by cell transplantation as a treatment option for CLI [4-6]. Cell-based therapeutic angiogenesis aims to improve limb perfusion by enhancing neovascularization using autologous bone marrow mononuclear cells (BMNCs), stem cells, endothelial progenitor cells (EPCs), and peripheral blood mononuclear cells (PBMNCs), thereby regulating both the secretion of proangiogenic factors and endothelial differentiation. Therapeutic angiogenesis is thought to be especially important for diabetic patients with PAD because there is no current treatment other than amputation for CLI in such patients. However, diabetes reduces the effectiveness of cell-based therapy [5], although the mechanisms underlying this interference remain unknown.

We recently reported the development of a biodegradable injectable cell scaffold (ICS), which is a nano-scaled hydroxyapatite (HAp)-coated poly(L-lactic acid) (PLLA) microsphere that enhances therapeutic angiogenesis [7]. HAp is the main 
mineral component of bones and teeth, and artificially synthesized HAp has been extensively used as a biomaterial because of its excellent adhesive properties not only to hard and soft tissues but also to various type of cells [8]. HAp nanocrystal coating was used for the ICS to overcome the disadvantage of low cell adhesion property of biodegradable polymer with hydrophobic surfaces [9]. Because about $80 \%$ of the transplanted cells disappear from the injection site within 1 week after transplantation of BMNCs alone, maintaining transplanted cells in place is crucial to augment the effectiveness of cell-based therapeutic angiogenesis. In fact, the ICS markedly enhances angiogenesis and arteriogenesis through cell clustering around the ICS, prolongs the presence of transplanted cells, augments expression of proangiogenic factors, and increases cell survival in non-diabetic mice [7]. However, because impaired proangiogenic potential of bone marrow-derived cells is known in diabetes [10], whether ICS is also useful in a diabetic model remains unknown. Its efficacy must be verified in a diabetic model.

Furthermore, in order for the ICS to be clinically applicable, modifications must be made to improve it according to clinical use. In order to appropriately accelerate absorption to avoid residual foreign particle accumulation in the living body, an improved version was made by replacing the biodegradable polymers constituting the core of the ICS from PLLA with a molecular weight half-life of 52 weeks to copolymer of lactic acid and $\varepsilon$-caprolactone (75:25 ratio) with a molecular weight half-life of 8 weeks, poly(L-lactide-co-e-caprolactone) (PLCL) (75.25) [11, 12]. PLCL is one of the most promising biodegradable polymers and has been used in the fields of orthopedic and reconstructive surgery and tissue engineering [12]. Furthermore, the particle diameter was reduced from $100 \mu \mathrm{m}$ to $50 \mu \mathrm{m}$ to facilitate injection of a mixed 
preparation of cells and ICS using a 24-22 gauge needle (an inner diameter of 370-480 $\mu \mathrm{m})$ and to prevent clogging during intramuscular injection into patients [11]. However, these modifications can hamper the function of the ICS, because too rapid absorption can reduce cell-retaining potential. Furthermore, smaller microspheres can exhibit less cell adhesiveness because of greater curvature of the surface. Thus, the effectiveness of the novel ICS must be confirmed.

In this study, the efficacy of the practical ICS was confirmed using an ischemic rabbit model, which is a larger-animal model than that of the mouse. Moreover, we examined whether ICS can augment the efficacy of therapeutic angiogenesis in mice in a diabetic state that is often accompanied by CLI and markedly inhibits the efficacy of therapeutic angiogenesis. 


\section{Materials and Methods}

\subsection{Animals}

BALB/cAJcl mice were purchased from Clea Japan, Inc. (Tokyo, Japan). Male New Zealand white rabbits, enhanced green fluorescent protein (EGFP)-transgenic C57/BL6-Tg (CAG-EGFP) mice, and wild-type C57BL/6NCrSlc mice were purchased from Japan SLC, Inc. (Hamamatsu, Japan). The procedures used in this study were approved by the Animal Care and Use Committee of Osaka City University Graduate School of Medicine, Osaka, Japan (approval number, 09023). Streptozotocin (STZ) induced insulin-deficient diabetic mice were prepared as previously described [13].

\subsection{Materials}

Rat monoclonal anti-vascular endothelial growth factor (VEGF) antibody (clone: RM0009-2G02) was purchased from Angio-Proteomie (Boston, MA), rabbit polyclonal anti-fibroblast growth factor-2 (FGF-2) antibody was obtained from Abcam (Cambridge, UK), and Alexa Fluor 594-conjugated goat anti-rat IgG antibody and Alexa Fluor 594-conjugated chicken anti-rabbit IgG antibody were purchased from Invitrogen Life Technologies (Carlsbad, CA). 4',6-Diamino-2-phenylindole dihydrochloride (DAPI) was obtained from Pierce Biotechnology (Rockford, IL). The GFP ELISA kit was obtained from Abcam (Cambridge, UK). E-Z TRAC Ultraspheres $^{\mathrm{TM}}$ (colored microspheres $100 \mu \mathrm{m}$ in diameter) was obtained from Interactive Medical Technologies, Ltd (Irvine, CA).

\subsection{Fabrication of novel ICS}

The ICS was prepared according to our previous report [11]. 


\subsection{Rabbit model of unilateral hindlimb ischemia and therapeutic angiogenesis by autologous BMNCs transplantation}

Neovascular formation in response to tissue ischemia was examined in a rabbit model of unilateral hindlimb ischemia as described elsewhere [14]. Briefly, at 1 week after femoral artery excision, animals were transplanted with autologous BMNCs with or without ICS as described below. Isolated autologous BMNCs from both iliac crests were suspended in $1 \mathrm{~mL}$ of serum-free Dulbecco's Modified Eagle's Medium and incubated with or without ICS at $37^{\circ} \mathrm{C}$ for $3 \mathrm{~h}$. Rabbits subjected to unilateral limb ischemia were randomly divided into three groups. The control group received $1 \mathrm{~mL}$ vehicle. The second group received autologous BMNCs $\left(3.5 \pm 1.1 \times 10^{7}\right.$ cells per animal; BMNCs group), and the third group received autologous BMNCs and the ICS $\left(3.8 \pm 0.9 \times 10^{7}\right.$ cells per animal; BMNCs + ICS group). One mg of the ICS was co-transplanted with $1.5 \times 10^{6}$ cells of BMNCs. At 2 weeks after transplantation, angiographic study or microvessel evaluating study were performed.

\subsection{Digital subtraction angiography and measurement of microvascular density}

To visualize dynamic perfusion, digital subtraction angiography (Phillips BV312, Philips, Amsterdam, The Netherlands) was performed as previously described [15]. To ensure consistency of imaging conditions of angiography pictures, we evaluated collateral vessels using pictures showing the full length of the caudal artery. Collateral vessel formation was quantitatively assessed using angiogram as previously described [16]. Microvascular density were also evaluated using colored microspheres in accordance with the manufacturer's instructions of E-Z TRAC Ultraspheres ${ }^{\mathrm{TM}}$ 
(Interactive Medical Technologies, Ltd, CA) and a previous report [17]. If needed, see the electronic supplementary material for further details.

\subsection{BMNC transplantation in ischemic hindlimb of mice}

BMNCs were harvested from 8-week-old male mice as previously described [7]. BMNCs $\left(5 \times 10^{6}\right.$ cells $)$ were intramuscularly injected, without or with a $1.5 \mathrm{mg}$ $(12,000$ particles $)$ of ICS, into the unilateral ischemic hindlimbs of 8 -week-old male mice immediately after operative resection of the artery and vein, as described elsewhere [7]. Diabetic or non-diabetic mice were implanted with BMNCs derived from diabetic or non-diabetic mice, respectively, without crossover. Ischemic operation, cell transplantation, and evaluation of limb necrosis were performed by separate operators in a blinded manner.

\subsection{Measurement of GFP levels in ischemic tissue}

We collected whole thigh muscle tissues 7 days after induction of limb ischemia. GFP levels of the tissue lysate were quantified as previously described [7].

\subsection{Immunohistochemistry and terminal deoxynucleotidyl transferase-mediated biotin-16-dUTP nick-end labelling (TUNEL) Assay}

Immunohistochemical staining for VEGF and FGF-2, and TUNEL assay in ischemic thigh tissue were performed as previously described [7].

\subsection{Statistical analysis}

Data are presented as means (SD). Statistical significance was evaluated by 
ANOVA and Tukey's test for comparison and contrast between multiple groups. Plots of the estimated limb survival ratio after the operation were constructed by the Kaplan-Meier method and were compared using the log-rank test. Odds ratios (ORs) of each predictor for limb necrosis were estimated by univariate logistic regression analysis. Statistical analyses were performed using JMP 10.0.2 software and STATVIEW 5.0 software (SAS Institute Inc., Cary, NC). P-values less than 0.05 were considered statistically significant.

\section{Results}

\subsection{Co-transplantation of ICS and BMNCs enhances collateral vessel formation in a rabbit model.}

We previously presented the enhancing effect of a prototype ICS on cell-based therapeutic angiogenesis using a mouse ischemic model [7]. In this study, we first confirmed the usefulness of a practical ICS using a rabbit model, which involves a much larger animal compared to the mouse model. An angiographic study was performed 3 weeks after ischemic induction (Fig. 1A). In order to examine changes in collateral vessels by therapeutic angiogenesis, a region of interest (ROI) was set at the central side of the popliteal artery stump, and the ratio of the vascular areas to the total area of ROI was calculated as an angiogenic index. No significant difference in angiogenic index was seen between the co-transplantation of ICSs and BMNCs (BMNCs + ICS group) and BMNCs only (BMNCs group); however, the angiogenic index was significantly higher in the BMNCs + ICS group, but not in the BMNCs group, as compared to the vehicle group (Fig. 1B). In addition, using colored microspheres, we examined the microvascular density in ischemic muscle that can trap microspheres of 
$100 \mu \mathrm{m}$ in diameter. The estimated microvascular density in the BMNCs + ICS group was markedly higher than in the vehicle group and the BMNCs group (Fig. 1C).

\subsection{Co-transplantation of the ICS and BMNCs enhances limb salvage even in}

\section{diabetic mice.}

Diabetic and non-diabetic mice were implanted with BMNCs from diabetic and non-diabetic mice, respectively, without crossover. Hindlimb ischemia in BALB/c mice was used as an intractable ischemia model as these mice show little spontaneous collateral vessel formation in response to ischemia with ischemic hindlimb necrosis [7]. In this limb ischemic model without diabetes, the BMNCs group showed better limb survival, while the BMNCs + ICS group revealed significantly more limb salvage than the BMNCs group (Fig. 2A). Injection of the ICS alone did not improve limb survival as shown previously in the prototype ICS [7] (data not shown). In diabetic mice, BMNCs + ICS improved limb survival significantly, while BMNCs alone showed little or no therapeutic effect (Fig. 2B).

Univariate logistic regression analysis was performed to investigate the impact of each intervention or the presence of diabetes on limb necrosis using the combined data of mice in Fig. 1A and B (Table 1). In non-diabetic mice, BMNC transplantation reduced the risk of limb necrosis by $67 \%$, while BMNCs + ICS reduced the risk by $83 \%$ compared to the vehicle group. In diabetic mice, BMNCs alone did not reduce the risk of limb necrosis. In contrast, BMNCs + ICS reduced the risk by $89 \%$. Moreover, we evaluated the impact of diabetes on each intervention. While diabetes had a 3.6-times higher risk of limb necrosis in the BMNCs group, diabetes did not have an effect in the BMNCs + ICS group. 


\subsection{ICS prevents apoptotic cell death of transplanted BMNCs in ischemic tissue of}

\section{diabetic mice.}

To evaluate the mechanism underling limb salvage enhancement by the ICS during $\mathrm{BMNC}$ transplantation in diabetic mice, we first investigated the localization of transplanted BMNCs in diabetic mice. BMNCs from EGFP-transgenic diabetic mice and ICSs were transplanted into the ischemic hindlimbs of C57BL/6NCrSlc diabetic mice. Intramuscular levels of GFP derived from BMNCs were significantly higher in the BMNCs + ICS group than in the BMNCs group at 7 days after transplantation both in non-diabetic and diabetic mice (Fig. 3A). GFP levels of the BMNCs and BMNCs + ICS group were not significantly different between non-diabetic and diabetic mice. Next, we investigated the impact of diabetes on apoptotic cell death of transplanted BMNCs. Ten days after injection of BMNCs alone, a larger proportion of transplanted BMNCs were positive for TUNEL, suggesting apoptotic cell death, in diabetic mice than in non-diabetic mice (Fig. 3B and C). In contrast, little TUNEL-positivity was observed in BMNCs around the ICS even in diabetic animals.

To clarify the mechanism underlying how the ICS prevents apoptosis of transplanted BMNCs, we investigated the localization of the proangiogenic mediators VEGF and FGF-2, also known as anti-apoptotic factors for transplanted BMNCs, in ischemic muscle of diabetic mice 7 days after transplantation. The expression of these factors mostly co-localized with implanted GFP-positive BMNCs around the ICS, with some diffusion from the clusters of BMNCs and ICS (Fig. 3D). 


\section{Discussion}

The present study demonstrated the effectiveness of a practical ICS in enhancing cell-based therapeutic angiogenesis in rabbits, which is an animal that is much larger than a mouse, and in STZ-induced diabetic mice. Furthermore, this is the first study to demonstrate that diabetes significantly accelerates apoptotic cell death of transplanted cells in vivo, compared to non-diabetic mice. The ICS provides a promising local environment for implanted cells with positive effects on limb salvage even in diabetic animals, similar to non-diabetic animals.

In this study, the transplantation of BMNCs alone decreased the risk of limb necrosis by $68 \%$ in non-diabetic animals, whereas the transplantation of BMNCs alone showed no therapeutic effect in diabetic animals (Table 1). Meanwhile, the limb salvage effect by co-transfection of the ICS and BMNCs was comparable in the diabetic model and the non-diabetic model (Fig. 2 and Table 1). The effectiveness of cell-based therapeutic angiogenesis is reportedly lower in diabetic patients than in non-diabetic patients [5], but the cause of this remains to be elucidated. Since local administration of healthy CD34-positive angiogenic cells improves blood flow and wound healing in hindlimb ischemia and skin wound models of diabetic mice $[18,19]$, alterations in implanted cells by a diabetic milieu may be responsible for the lower therapeutic efficacy in diabetic subjects. EPCs are known as primary bone marrow-derived angiogenesis-promoting cells. EPCs isolated from a diabetic model reportedly have the decreased ability to secrete cytokine and to migrate, and promote apoptosis in vitro [10, 20]. This study also showed that apoptotic cell death is higher in transplanted BMNCs in diabetic animals than in non-diabetic animals in vivo (Fig. 3B). We previously reported that the therapeutic benefit of cell-based therapeutic angiogenesis using the 
ICS is attributable to an antiapoptotic effect against transplanted cells [7]. In this study, ICS and BMNC co-translation inhibited apoptosis of BMNCs in diabetic animals similarly to the effects seen in non-diabetic animals (Fig. 3C), suggesting that the decrease in apoptosis of transplanted cells results in favorable limb salvage in the diabetic model.

Moreover, reduced cytokine secretion from transplanted cells may impede the effects of cell-based therapy in diabetic patients. Reduced production of VEGF is reported to cause lower limb necrosis in ischemic lower limb tissues [20, 21]. As VEGF production is reportedly reduced in BMNCs isolated from diabetic rats [22], insufficient VEGF activity in the local transplanted site is likely responsible for the reduced efficacy of cell-based therapy in diabetic subjects. We previously reported that VEGF and FGF-2 are continuously secreted from aggregates of the ICS and transplanted cells and that their tissue levels increase in non-diabetic animals [7]. Meanwhile, in this study using a diabetic model, co-transplantation of BMNCs and ICS did not result in an increase in the mean cytokine level in ischemic muscle (data not shown). Thus, the capacity of cytokine secretion from BMNCs decreased in diabetic animals. However, a histopathological examination confirmed strongly localized VEGF and FGF-2 in aggregates of ICS and BMNCs in diabetic animals (Fig. 3D), which may indicate that a continuous increase in cytokine level at the local transplant site, but not an increase in the entire ischemic muscle, is important for inducing the limb salvage effect.

Issues remain in the clinical application of the ICS. First, the efficacy of the ICS must be confirmed in the presence of angiogenesis inhibitory factors other than diabetes. For example, we previously reported that end-stage renal disease (ESRD) markedly inhibits the efficacy of cell-based therapeutic angiogenesis [4]. The cause of 
cell-therapy inhibition by ESRD has not been fully elucidated; however, lower EPC abundance and impaired angiogenic function of EPCs are associated with dialysis efficiency [23]. Therefore, EPC disorders induced by uremic toxin may be at least partly responsible for angiogenesis inhibition in ESRD patients. Whether the ICS is effective in patients with decreased angiogenesis caused by uremic toxin must be investigated. Next, further studies are needed to evaluate differences in efficacy of the ICS according to type of transplanted cells. For example, during PBMNC transplantation, angiogenic factors, such as interleukin-1beta, were secreted from muscle cells activated through interaction with transplanted cells, rather than cytokines secreted from transplanted cells [24]. As discussed, the mechanism of angiogenesis induction may vary by type of transplanted cells; therefore, the effectiveness of the ICS requires confirmation in a cell and tissue-specific manner.

In conclusion, the practical ICS significantly augmented cell-based therapeutic angiogenesis even in diabetic animals, through local accumulation of proangiogenic factors and antiapoptotic effects of transplanted cells.

\section{Acknowledgements}

We are grateful to Ms. Chiaki Murakami, Osaka City University Graduate School of Medicine, for her technical assistance. This study was supported in part by the Adaptable and Seamless Technology Transfer Program through target-driven R\&D (A-STEP) by the Japan Science and Technology Agency (JST) (AS2314097F to S.F.). 


\section{References}

[1] L. Norgren, W.R. Hiatt, J.A. Dormandy, et al., Inter-Society Consensus for the Management of Peripheral Arterial Disease (TASC II), Journal of vascular surgery : official publication, the Society for Vascular Surgery [and] International Society for Cardiovascular Surgery, North American Chapter, 45 Suppl S (2007) S5-67.

[2] P.K. Moulik, R. Mtonga, G.V. Gill, Amputation and mortality in new-onset diabetic foot ulcers stratified by etiology, Diabetes care, 26 (2003) 491-494.

[3] L. Graziani, A. Silvestro, V. Bertone, et al., Vascular involvement in diabetic subjects with ischemic foot ulcer: a new morphologic categorization of disease severity, European journal of vascular and endovascular surgery : the official journal of the European Society for Vascular Surgery, 33 (2007) 453-460.

[4] S. Matoba, T. Tatsumi, T. Murohara, et al., Long-term clinical outcome after intramuscular implantation of bone marrow mononuclear cells (Therapeutic Angiogenesis by Cell Transplantation [TACT] trial) in patients with chronic limb ischemia, American heart journal, 156 (2008) 1010-1018.

[5] Z. Raval, D.W. Losordo, Cell therapy of peripheral arterial disease: from experimental findings to clinical trials, Circulation research, 112 (2013) 1288-1302.

[6] M. Teraa, R.W. Sprengers, Y. van der Graaf, et al., Autologous bone marrow-derived cell therapy in patients with critical limb ischemia: a meta-analysis of randomized controlled clinical trials, Annals of surgery, 258 (2013) 922-929.

[7] Y. Mima, S. Fukumoto, H. Koyama, et al., Enhancement of cell-based therapeutic angiogenesis using a novel type of injectable scaffolds of hydroxyapatite-polymer nanocomposite microspheres, PloS one, 7 (2012) e35199.

[8] H. Aoki, Medical Applications of Hydroxyapatite, Ishiyaku EuroAmerica, St. Louis, 
1994.

[9] S. Fujii, M. Okada, H. Sawa, et al., Hydroxyapatite nanoparticles as particulate emulsifier: fabrication of hydroxyapatite-coated biodegradable microspheres, Langmuir : the ACS journal of surfaces and colloids, 25 (2009) 9759-9766.

[10] K.Y. Howangyin, J.S. Silvestre, Diabetes mellitus and ischemic diseases:

molecular mechanisms of vascular repair dysfunction, Arteriosclerosis, thrombosis, and vascular biology, 34 (2014) 1126-1135.

[11] S. Fujii, Y. Miyanari, T. Nishimura, et al., In vitro degradation of hydroxyapatite nanoparticle-coated biodegradable microspheres, Polymer Degradation and Stability, 98 (2013) 377-386.

[12] Y. Naito, T. Shinoka, D. Duncan, et al., Vascular tissue engineering: towards the next generation vascular grafts, Advanced drug delivery reviews, 63 (2011) 312-323. [13] T. Shoji, H. Koyama, T. Morioka, et al., Receptor for advanced glycation end products is involved in impaired angiogenic response in diabetes, Diabetes, 55 (2006) $2245-2255$.

[14] S. Shintani, T. Murohara, H. Ikeda, et al., Augmentation of postnatal neovascularization with autologous bone marrow transplantation, Circulation, 103 (2001) 897-903.

[15] Y. Sakai, R. Yamada, K. Nakamura, et al., Angiographic and histologic changes in artery, vein and portal vein after placement of bare or covered Z-stent; an experimental study in dogs, Osaka city medical journal, 47 (2001) 43-52.

[16] A. Seko, N. Nitta, A. Sonoda, et al., Vascular regeneration by repeated infusions of basic fibroblast growth factor in a rabbit model of hind-limb ischemia, AJR. American journal of roentgenology, 192 (2009) W306-310. 
[17] S. Takeshita, T. Isshiki, M. Ochiai, et al., Endothelium-dependent relaxation of collateral microvessels after intramuscular gene transfer of vascular endothelial growth factor in a rat model of hindlimb ischemia, Circulation, 98 (1998) 1261-1263.

[18] G.C. Schatteman, H.D. Hanlon, C. Jiao, et al., Blood-derived angioblasts accelerate blood-flow restoration in diabetic mice, The Journal of clinical investigation, 106 (2000) 571-578.

[19] E. Sivan-Loukianova, O.A. Awad, V. Stepanovic, et al., CD34+ blood cells accelerate vascularization and healing of diabetic mouse skin wounds, Journal of vascular research, 40 (2003) 368-377.

[20] P.Z. Costa, R. Soares, Neovascularization in diabetes and its complications. Unraveling the angiogenic paradox, Life sciences, 92 (2013) 1037-1045.

[21] T.G. Ebrahimian, R. Tamarat, M. Clergue, et al., Dual effect of angiotensin-converting enzyme inhibition on angiogenesis in type 1 diabetic mice, Arteriosclerosis, thrombosis, and vascular biology, 25 (2005) 65-70.

[22] T.S. Li, A. Furutani, M. Takahashi, et al., Impaired potency of bone marrow mononuclear cells for inducing therapeutic angiogenesis in obese diabetic rats, American journal of physiology. Heart and circulatory physiology, 290 (2006) H1362-1369.

[23] J.H. Choi, K.L. Kim, W. Huh, et al., Decreased number and impaired angiogenic function of endothelial progenitor cells in patients with chronic renal failure, Arteriosclerosis, thrombosis, and vascular biology, 24 (2004) 1246-1252.

[24] K. Tateno, T. Minamino, H. Toko, et al., Critical roles of muscle-secreted angiogenic factors in therapeutic neovascularization, Circulation research, 98 (2006) 1194-1202. 


\section{Figure legends}

\section{Figure 1}

\section{Collateral vessel formation in rabbits with hindlimb ischemia by autologous}

BMNC transplantation with ICS.

(A) Digital subtraction angiography was performed at 2 weeks after transplantation of vehicle, BMNCs alone, or BMNCs + ICS. Representative angiograms of left ischemic lower limbs are presented. (B) Quantitative analysis of collateral vessels in the ischemic thigh area were performed using the arterial phase digital subtraction angiogram data (vehicle, $\mathrm{n}=3$; BMNCs alone, $\mathrm{n}=3$; BMNCs + ICS, $\mathrm{n}=4$ ). (C) Quantitative analysis for microvascular density using colored microspheres $100 \mu \mathrm{m}$ in diameter was also performed at 2 weeks after transplantation of vehicle, BMNCs alone, or BMNCs + ICS ( $\mathrm{n}=4$ in each group). Data are shown as means (SD). ${ }^{*}, P<0.05$. Abbreviations: ICS, injectable cell scaffolds; BMNC, bone marrow mononuclear cell.

\section{Figure 2}

\section{Augmentation of limb salvage by co-transplantation of ICS and BMNCs in}

\section{diabetic mice.}

The survival curve for limb necrosis of hindlimb ischemic mice after ischemic induction and simultaneous intramuscular transplantation of vehicle, BMNCs alone, or BMNCs + ICS. Non-diabetic and diabetic mice were transplanted with BMNCs from non-diabetic (A) and diabetic mice (B), respectively, without crossover. Limb necrosis was evaluated every day after the ischemic operation in a blinded manner. The curve was obtained using the Kaplan-Mayer method, and the difference between the two groups was compared using the log-rank test. ${ }^{*}, P<0.05$. Abbreviations: ICS, injectable cell 
scaffold; BMNC, bone marrow mononuclear cell.

\section{Figure 3}

\section{Impact of concomitant ICS use to transplanted BMNCs in diabetic mice.}

(A) Quantitative evaluation of transplanted cells sustained in ischemic tissues. Murine BMNCs derived from EGFP-transgenic mice without or with diabetes were transplanted into the thighs in the hindlimb ischemic model without or with diabetes, respectively. Quantitative analysis of intramuscular GFP was performed 7 days after transplantation. BMNCs were transplanted alone or together with the ICS. Intramuscular GFP values in whole thigh muscles were corrected for total protein and expressed in arbitrary units (n $=3$ in each group). (B) ICS prevention against apoptotic cell death of transplanted cells in diabetic mice. BMNCs (green) were derived from EGFP-transgenic diabetic mice. Tissue sections from hindlimb ischemic mice with diabetes 10 days after transplantation were counterstained with DAPI (blue). Apoptotic nuclei were stained with tetramethylrhodamine (TMR) (red) by the TUNEL method. Scale bars, $50 \mu \mathrm{m}$. (C) The percentage of TUNEL-positive cells compared to GFP-positive ones was assessed in four low-power fields in each mouse ( $\mathrm{n}=3$ in each group). (D) Colocalization of transplanted BMNCs and proangiogenic factors, VEGF (upper panel) and FGF-2 (lower panel), around ICSs. Tissue sections from hindlimb ischemic mice with diabetes 7 days after transplantation of BMNCs + ICS were immunofluorescently stained using anti-mouse VEGF antibody or anti-mouse FGF-2 antibody and DAPI. BMNCs were derived from EGFP-transgenic mice. Scale bars, $50 \mu \mathrm{m}$.

Data are shown as means (SD). ${ }^{*}, P<0.05$. Abbreviations: DM, diabetes mellitus; ICS, injectable cell scaffold; BMNC, bone marrow mononuclear cell; GFP, green fluorescent 
protein; DAPI, 4',6-diamidino-2-phenylindole; TUNEL, terminal deoxynucleotidyl transferase-mediated dUTP-biotin nick-end labelling.

\section{Table legend}

\section{Table 1.}

\section{The impact of each predictor for limb necrosis in each stratified group}

The odds ratios and 95\% confidence interval (CI) of each predictor for limb necrosis estimated by univariate logistic regression analysis in each stratified group according to diabetic conditions or interventions are shown. Diabetes and intervention were represented by dummy nominal variables $($ Non $\mathrm{DM}=0, \mathrm{DM}=1 ;$ Vehicle $=0$, BMNCs $=1$, BMNCs + ICS = 2). Abbreviations: CI, confidence interval; BMNCs, bone marrow mononuclear cells; ICS, injectable cell scaffold; DM, diabetes mellitus. 
Table 1. The impact of each predictor for limb necrosis in each stratified group

\begin{tabular}{|c|c|c|c|}
\hline \multirow[b]{2}{*}{ Stratification } & \multicolumn{3}{|c|}{ Odds ratio } \\
\hline & Predictors/reference & $(95 \% \mathrm{CI})$ & $\mathrm{p}$ \\
\hline \multirow{3}{*}{$\begin{array}{l}\text { Non-DM } \\
(\mathrm{N}=120)\end{array}$} & BMNCs/vehicle & $\begin{array}{c}0.321 \\
(0.118-0.826)\end{array}$ & 0.0181 \\
\hline & BMNCs + ICS/vehicle & $\begin{array}{c}0.174 \\
(0.063-0.450)\end{array}$ & 0.0002 \\
\hline & BMNCs + ICS/BMNCs & $\begin{array}{c}0.543 \\
(0.219-1.315)\end{array}$ & 0.1767 \\
\hline \multirow{3}{*}{$\begin{array}{l}\mathrm{DM} \\
(\mathrm{N}=60)\end{array}$} & BMNCs/vehicle & $\begin{array}{c}0.444 \\
(0.056-2.597)\end{array}$ & 0.3719 \\
\hline & BMNCs + ICS/vehicle & $\begin{array}{c}0.111 \\
(0.015-0.524)\end{array}$ & 0.0043 \\
\hline & $\mathrm{BMNCs}+\mathrm{ICS} / \mathrm{BMNCs}$ & $\begin{array}{c}0.250 \\
(0.056-0.965)\end{array}$ & 0.0441 \\
\hline $\begin{array}{l}\text { Vehicle } \\
(\mathrm{N}=60)\end{array}$ & DM/non-DM & $\begin{array}{c}2.613 \\
(0.590-18.391)\end{array}$ & 0.2187 \\
\hline $\begin{array}{l}\text { BMNCs } \\
(\mathrm{N}=60)\end{array}$ & DM/non-DM & $\begin{array}{c}3.619 \\
(1.100-14.375)\end{array}$ & 0.0336 \\
\hline $\begin{array}{l}\text { BMNCs + ICS } \\
(\mathrm{N}=60)\end{array}$ & DM/non-DM & $\begin{array}{c}1.667 \\
(0.561-5.006)\end{array}$ & 0.3559 \\
\hline
\end{tabular}




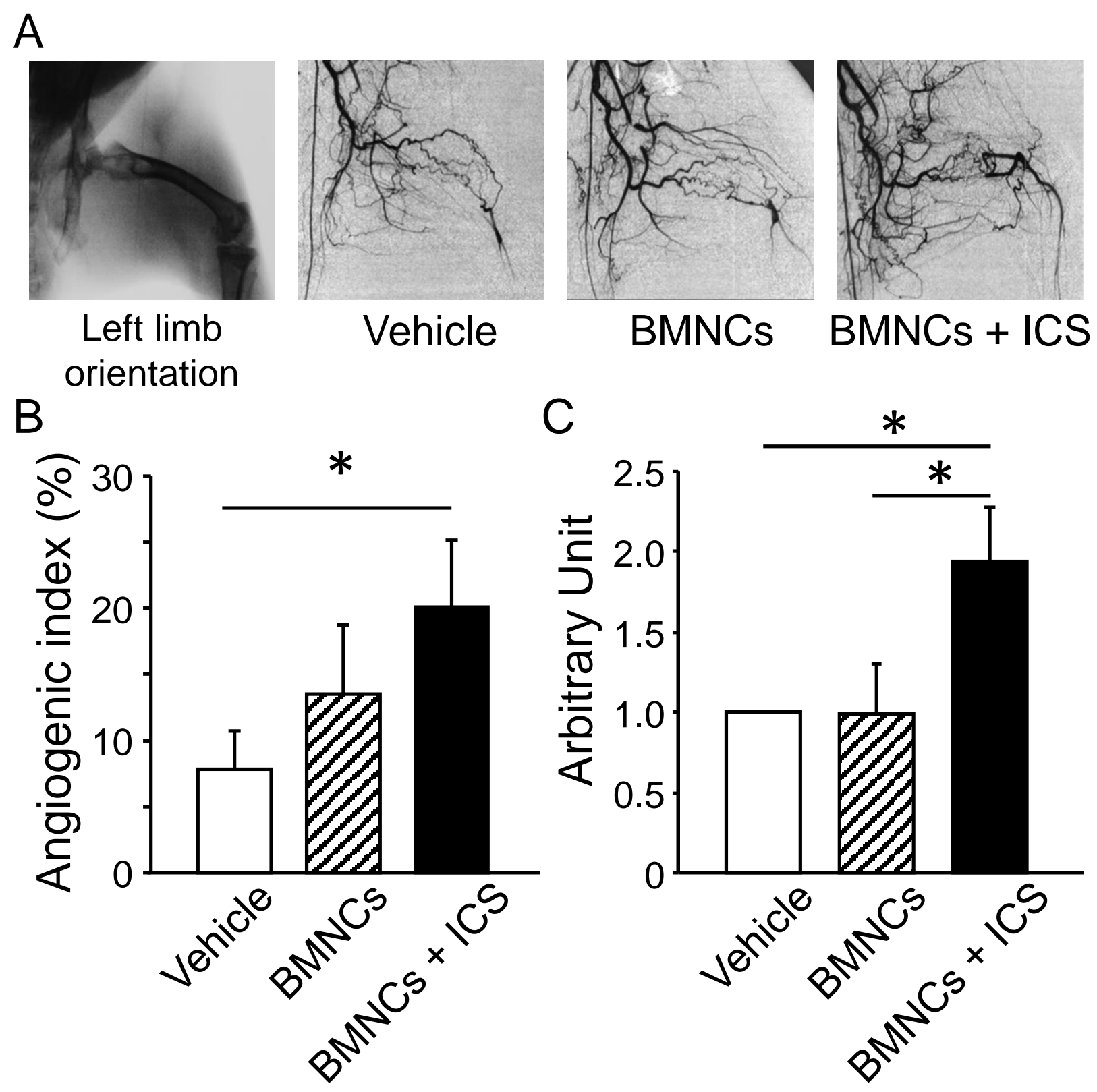



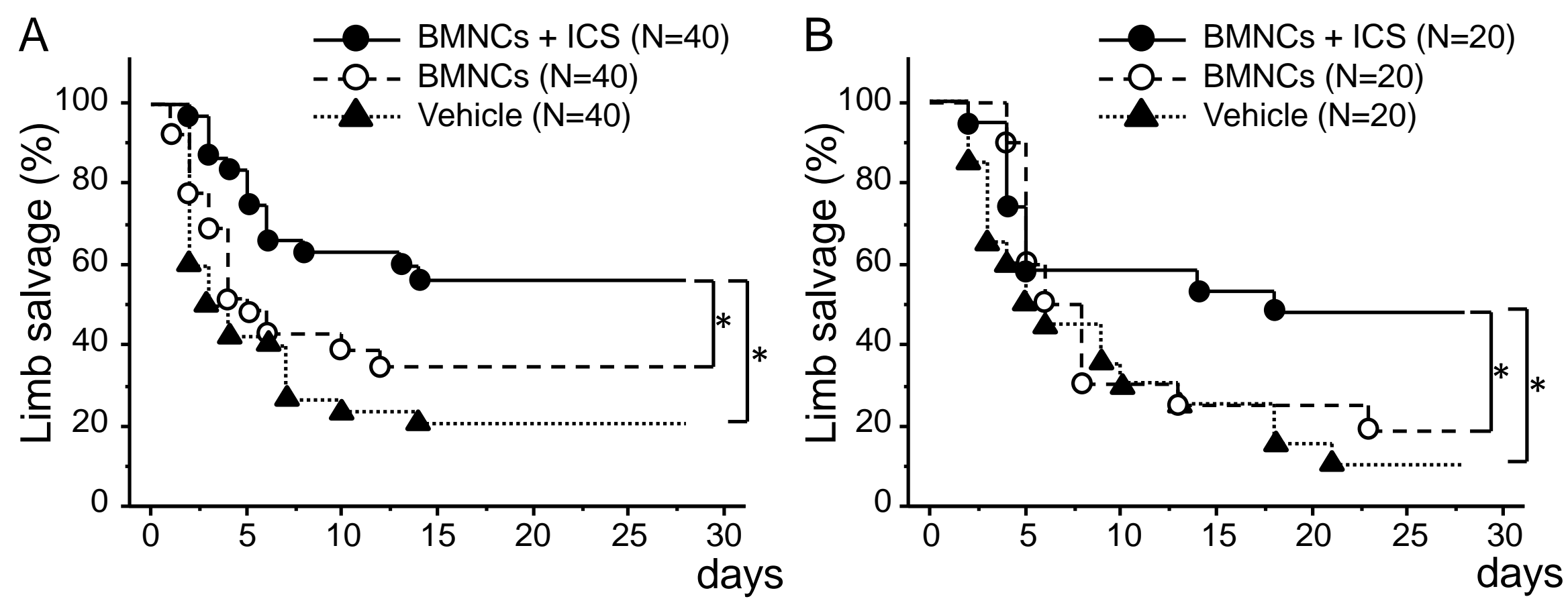

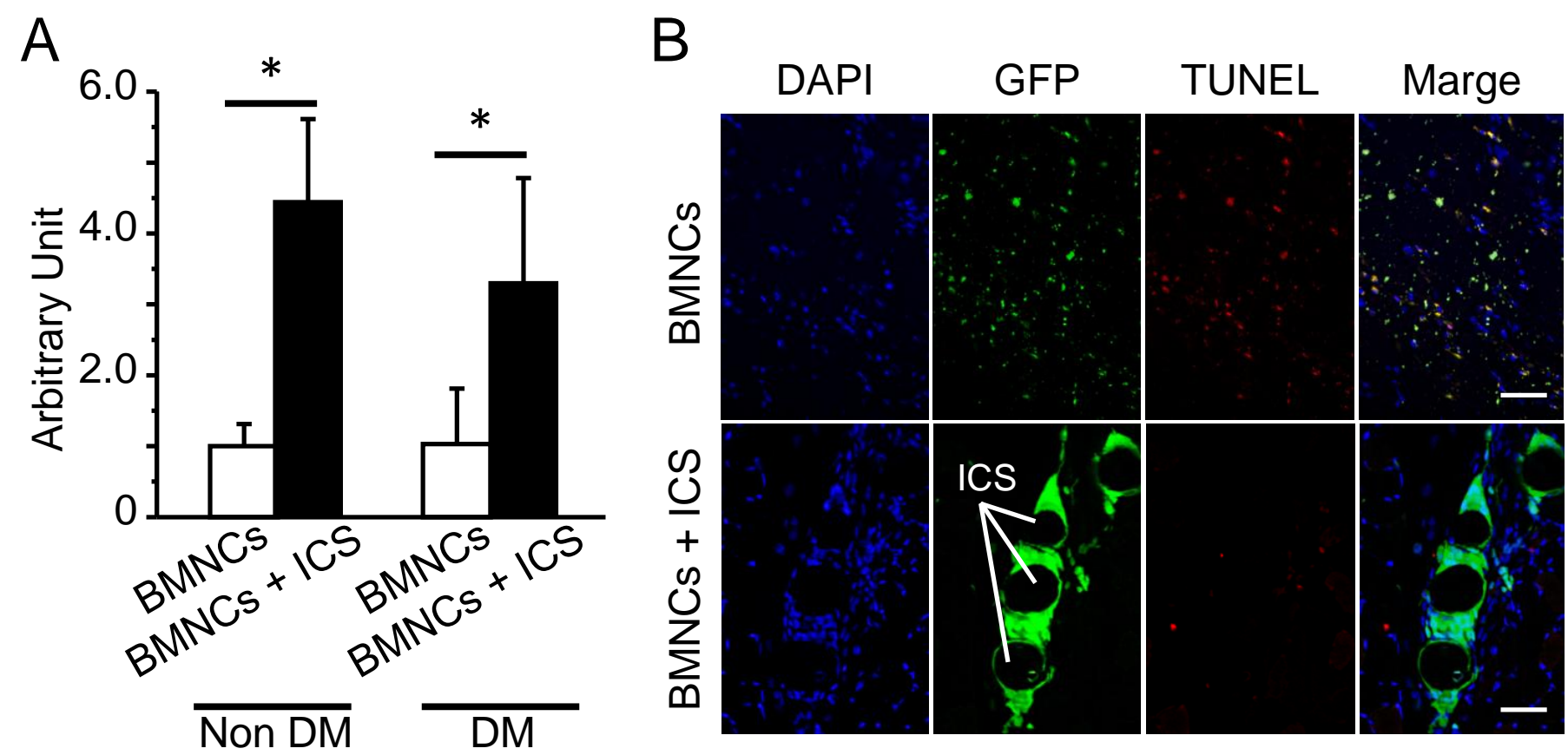

C

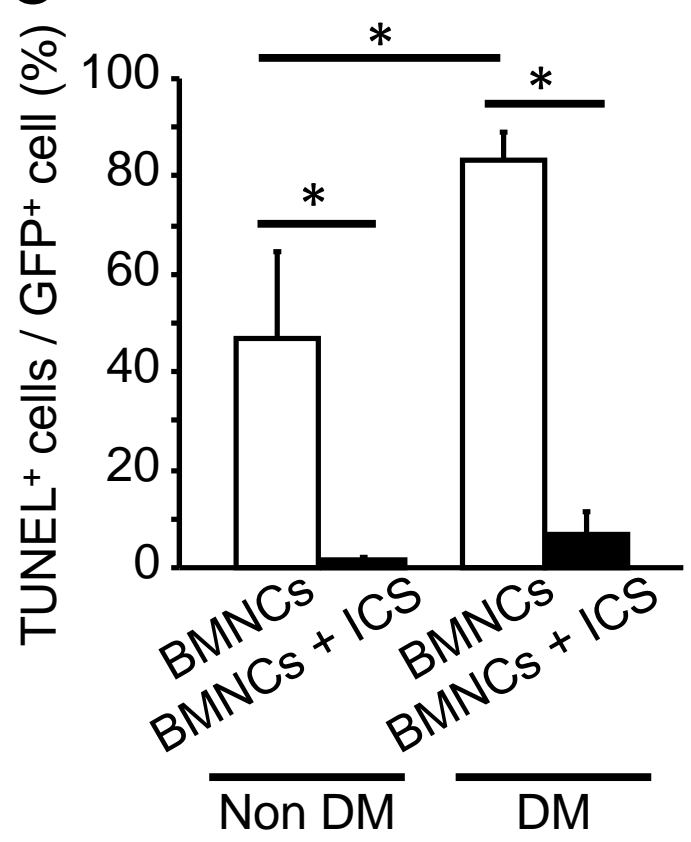

D

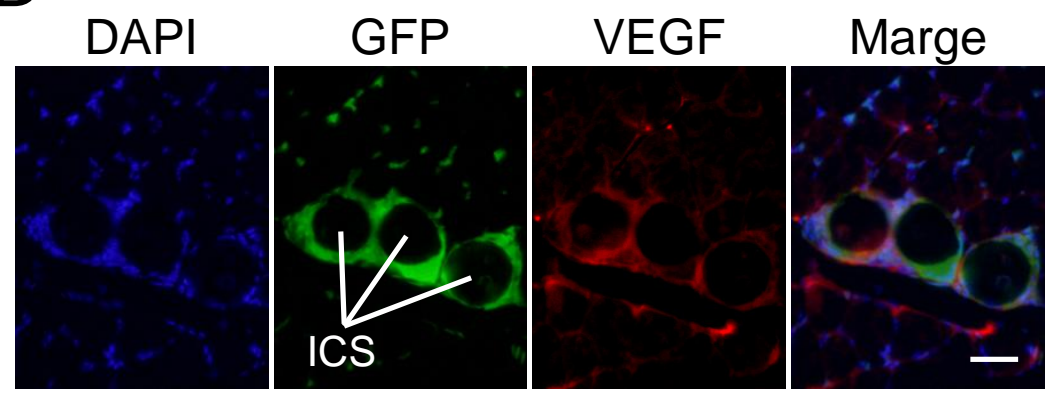

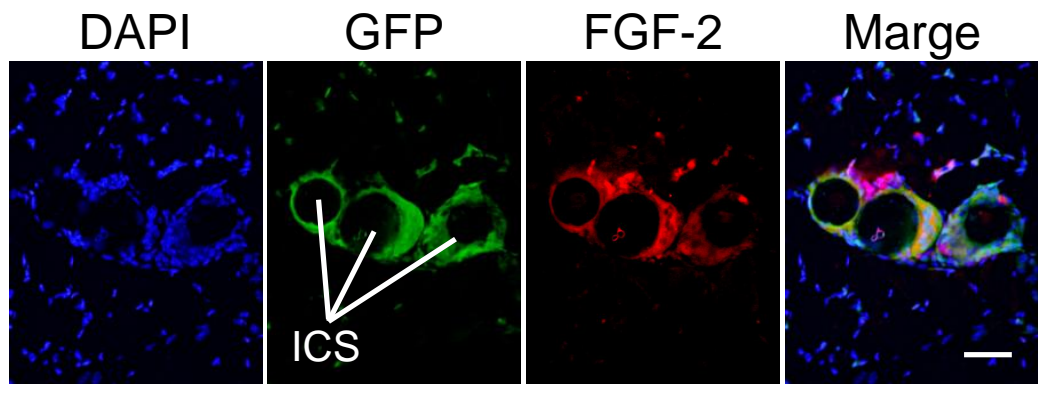

FGF-2
Marge 